\title{
The striatum specifies the statistics of behavior
}

\author{
Jeffrey E. Markowitz ${ }^{1}$ and Sandeep Robert Datta ${ }^{1}$ \\ Neuropsychopharmacology (2020) 45:222-223; https://doi.org/10.1038/s41386-019-0493-6
}

Many neurodegenerative disorders with motor phenotypes (e.g. Parkinson's and Huntington's disease) target an evolutionarily conserved, subcortical group of nuclei called the basal ganglia (BG). The input nucleus to this circuit is the largest structure in the BG, the striatum. Over $95 \%$ of the cells in the striatum are a unique type of GABAergic principal cell, the spiny projection neuron (SPN), which samples inputs from cortex and relays these signals to the rest of the BG. Because of the intimate relationship between $B G$ and behavior, understanding the physiological function of the BG-and how those functions are altered during diseaserequires the ability to carefully characterize behavior. Prior work on BG function in both humans and animals has relied on manual scoring of video recordings. The use of these methods has led to a model in which the BG initiates or suppresses broad classes of movement [1]. Specifically, SPNs expressing the DRD1 receptor (direct SPNs, or dSPNs), which contain a direct projection to the output of the BG, are thought to promote movements, while SPNs expressing the DRD2 receptor are thought to suppress them (indirect SPNs, or iSPNs).

Figure 1 Recently, our group has developed a machine learningbased approach called Motion Sequencing to better define the structure of ongoing behavior. In the context of the BG, these (and related) experiments have revealed that SPNs do not simply initiate or suppress broad classes of movements; instead, SPNs both reflect the content of individual movements (i.e., behavioral "syllables") and directly control how movements are sequentially organized (i.e., behavioral "grammar") [2,3].

Activity measurements from single dSPNs and iSPNs in freely moving mice demonstrated that both SPN types contain a "map" of syllables [4]. Similar populations of SPNs were active during similar syllables, and different neurons were active for dissimilar syllables. In our work, activity of dSPNs and iSPNs was monitored simultaneously, while also measuring exploratory mouse movements in 3D. We found that dSPNs and iSPNs encode action through a complementary code: more information about the animal's ongoing behavior is contained in both dSPN and iSPN activity compared with either in isolation [2] (see also [3]). Similar to [4], we found that the ensembles of dSPNs and iSPNs associated with any given behavior overlapped with those recruited during similar behaviors. Neurons active during a rear up in the center of the arena were likelier to be active during other behaviors with similar pose dynamics, for example rears along the wall or curved rears leading into a turn. Thus, the activity of SPNs could be directly mapped onto the landscape of pose dynamics between different behaviors. Furthermore, chemical lesion of the dorsolateral portion of the striatum led to aberrant sequencing of both spontaneous movements and sensory-guided movements. However, the execution of individual syllables was spared, similar to observations made in the context of grooming sequences in the rat [5].

Taken together, new recording technologies and automated methods for annotating behavior allowed our group, and others, to show that individual SPNs contain a relational map of action, dSPNs and iSPNs encode action complementarily, and SPNs control how movements are organized sequentially over time. This work reveals a more general role for both dSPNs and iSPNs in the process of selecting which action to perform among dozens of possible actions on a moment-by-moment basis. Thus, clinically, pathologies of the BG may be manifest both in the form and kinematics of movements and in the statistics of how movements are deployed over time.

\section{FUNDING AND DISCLOSURE}

SRD is supported by the Simons Collaboration on the Global Brain, NIH grants U24NS109520 and RO1DC016222, and by the Vallee Foundation. JEM is supported by a Burroughs Wellcome Foundation Career Award at the Scientific Interface. SRD is a cofounder of Syllable Life Sciences, Inc.

\section{CODE AVAILABILITY}

Details on how to obtain open-source code for the automated analysis of behavior are available at http://datta.hms.harvard.edu/research/behavioral-analysis/.

${ }^{1}$ Harvard Medical School, Department of Neurobiology, Boston, MA, USA

Received: 16 July 2019 Revised: 9 August 2019 Accepted: 14 August 2019

Published online: 29 August 2019 
Collect 3D depth videos of freely behaving mice

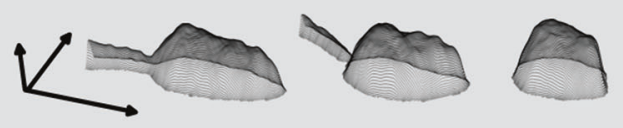

Automatically identify actions and transitions

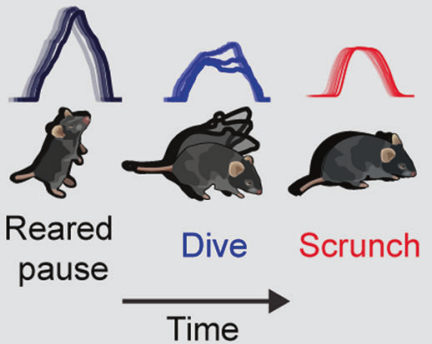

Align actions to neural activity

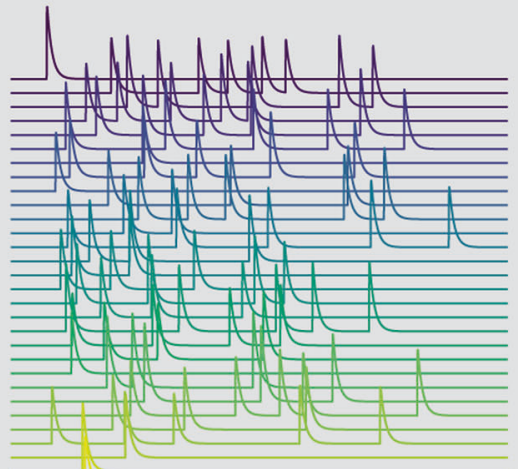

$\left|\begin{array}{c}\text { Reared } \\ \text { pause }\end{array}\right|$ Dive $\mid$ Scrunch $\mid$

Striatal neurons encode action content and sequence statistics

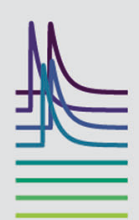

Action A

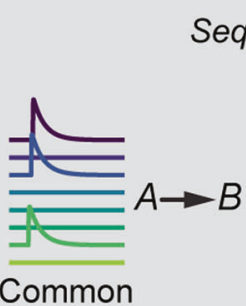

Actions
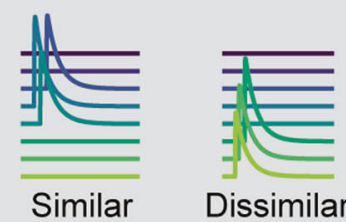

Dissimilar

Sequences

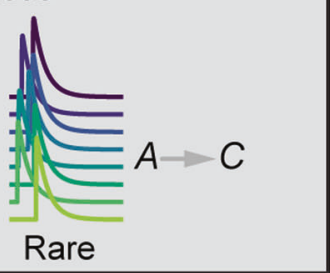

Lesions of the striatum disrupt action sequencing

Normal action sequence

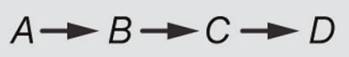
Lesion

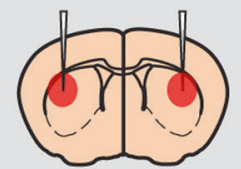

Disrupted action sequence

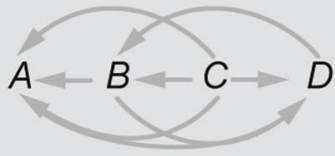

Fig. 1 Top, Schematic of the experimental workflow used in [2]. First, depth videos are collected from freely behaving mice from while simultaneously recording neural activity from SPNs. Then, behaviors and transitions are automatically identified using machine learning methods previously developed by our group [6]. Bottom left, Simultaneous measurement of 3D behavior and SPN activity revealed that SPNs encode the content and sequence structure of actions. Bottom right, Lesion experiments demonstrated that the striatum specifies how behaviors are sequenced

\section{ADDITIONAL INFORMATION}

Publisher's note: Springer Nature remains neutral with regard to jurisdictional claims in published maps and institutional affiliations.

\section{REFERENCES}

1. Kravitz AV, Benjamin SF, Parker PRL, Kay K, Thwin MT, Deisseroth K, et al. Regulation of parkinsonian motor behaviours by optogenetic control of basal ganglia circuitry. Nature. 2010;466:622-26.

2. Markowitz JE, Winthrop FG, Beron CC, Neufeld SQ, Robertson K, Bhagat ND, et al. The striatum organizes 3D behavior via moment-to-moment action selection. Cell. 2018;174:44-58.e17.
3. Meng C, Zhou J, Papaneri A, Peddada T, Xu K, Cui G. Spectrally resolved fiber photometry for multi-component analysis of brain circuits. Neuron. 2018;98:707-17.e4.

4. Klaus A, Gabriela JM, Paixao VB, Zhou P, Paninski L, Costa RM. The spatiotemporal organization of the striatum encodes action space. Neuron. 2017; 95:1171-80.e7.

5. Cromwell HC, Berridge KC. Implementation of action sequences by a neostriatal site: a lesion mapping study of grooming syntax. J Neurosci. 1996; 16:3444-58.

6. Wiltschko $A B$, Matthew JJ, lurilli G, Peterson RE, Katon JM, Pashkovski $\mathrm{SL}$, et al. Mapping sub-second structure in mouse behavior. Neuron. 2015;88:1121-35. 